Discrete Comput Geom 37:587-599 (2007)

DOI: $10.1007 / \mathrm{s} 00454-007-1308-9$

\title{
Staircase Connected Sets
}

\author{
Evelyn Magazanik and Micha A. Perles
}

Einstein Institute of Mathematics,

The Hebrew University of Jerusalem,

Jerusalem 91904, Israel

\{emaga,perles\}@math.huji.ac.il

\begin{abstract}
A compact set $S \subset \mathbb{R}^{2}$ is staircase connected if every two points $a, b \in S$ can be connected by a polygonal path with sides parallel to the coordinate axes, which is both $x$-monotone and $y$-monotone. $\xi(a, b)$ denotes the smallest number of edges of such a path. $\xi(\cdot, \cdot)$ is an integer-valued metric on $S$. We investigate this metric and introduce stars and kernels. Our main result is that the $r$ th kernel is nonempty, compact and staircase connected provided $r \geq \frac{1}{2} \cdot \operatorname{stdiam}(S)+1$.
\end{abstract}

\section{Staircase Sets}

Call a polygonal path $P=\left\langle a_{0}, a_{1}, \ldots, a_{n}\right\rangle$ in $\mathbb{R}^{2}$ orthogonal if every edge of $P$ is parallel to one of the coordinate axes (either horizontal or vertical). An orthogonal path $P$ is a staircase if all its horizontal edges point in the same direction (east or west) and all its vertical edges point in the same direction (south or north).

A set $S \subset \mathbb{R}^{2}$ is orthogonally connected (staircase connected) if $S \neq \emptyset$ and every two points $p, q \in S$ can be joined within $S$ by an orthogonal path (staircase). Note that if the segment $[p, q]$ is horizontal or vertical, then $[p, q]$ is the unique staircase that connects $p$ and $q$.

A set $S \subset \mathbb{R}^{2}$ is horizontally convex (vertically convex) if $S$ includes every horizontal (vertical) segment with endpoints in $S$. If $S$ is both horizontally and vertically convex, then we say that $S$ is orthogonally convex.

Proposition 1.1. A set $S \subset \mathbb{R}^{2}$ is staircase connected if and only if $S$ is orthogonally connected and orthogonally convex. 




Fig. 1. Shortcut.

Proof. The "only if" part is obvious. To prove the converse, consider two points $a, b \in S$ and let $P=\left\langle a, x_{1}, x_{2}, \ldots, x_{t-1}, b\right\rangle$ be an orthogonal path in $S$ from $a$ to $b$ with the smallest possible number of edges. If $P$ is not a staircase, then $P$ has two consecutive horizontal (or vertical) edges $\left[x_{i-1}, x_{i}\right]$ and $\left[x_{i+1}, x_{i+2}\right]$ with opposite directions. Since $S$ is orthogonally convex, $P$ can be replaced by a shorter orthogonal path within $S$, contradicting our initial choice of $P$. (See Fig. 1.)

Definition. A set $S \subset \mathbb{R}^{2}$ is called an $S_{k}$-set if every two points $a, b \in S$ can be joined within $S$ by a staircase with $k$ or fewer edges. We define the staircase diameter of $S$ $(\operatorname{stdiam}(S))$ to be the smallest $k$ such that $S$ is an $S_{k}$-set.

Proposition 1.2. Suppose $S \subset \mathbb{R}^{2}$ is compact and staircase connected. Then $S$ is an $S_{k}$-set for some finite $k$.

Proof. Let $L_{1}, L_{2}$ be the two vertical supporting lines of $S$ ( $L_{1}$ from the left, $L_{2}$ from the right). If $L_{1}=L_{2}$ then $S$ is a line segment (or a single point) and there is nothing to prove. So we may assume $L_{1}$ and $L_{2}$ are parallel. Take two points $c \in L_{1} \cap S$ and $d \in L_{2} \cap S$. Join $c$ and $d$ by a staircase $P=\left\langle c, x_{1}, x_{2}, \ldots, x_{k-1}, d\right\rangle$ within $S$. Given any two points $a, b \in S$ we can join $a$ to a point $a^{\prime} \in P$ and $b$ to a point $b^{\prime} \in P$ by vertical segments within $S$ ( $S$ is vertically convex), and obtain an orthogonal path $\left\langle a, a^{\prime}, \ldots, b^{\prime}, b\right\rangle$ with at most $k+2$ edges joining $a$ to $b$ within $S$. By Proposition 1.1 there is a staircase with $k+2$ or fewer edges that joins $a$ and $b$ within $S$.

Remarks. 1. Compactness of $S$ is necessary in Proposition 1.2 (see the example in Fig. 2):

$S=\left\{(\gamma, \eta) \in \mathbb{R}^{2}:|\gamma|<1,|\eta|<1,(\gamma+1)^{2}+(\eta-1)^{2} \leq 4,(\gamma-1)^{2}+(\eta+1)^{2} \leq 4\right\}$.

2. The analogue of Proposition 1.2 for polygonally connected sets, and even for orthogonally connected sets, is false, as Fig. 3 shows.

\section{The Staircase Distance}

Definition. Let $S \subset \mathbb{R}^{2}$ be a compact and staircase connected set, and let $a, b$ be two points in $S$. We denote by $\mu(a, b)$ a staircase path with the minimal number of edges 


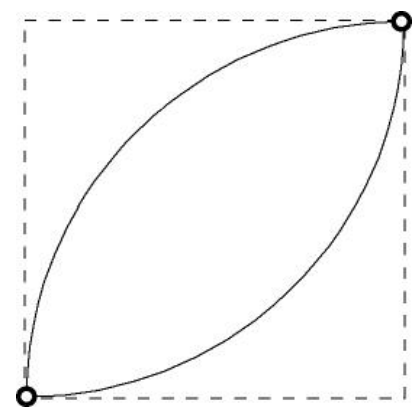

Fig. 2. A noncompact staircase connected set with infinite diameter.

connecting $a$ and $b$ within $S$. The number of edges of any polygonal path $P$ will be denoted by $e(P)$. Finally define $\xi(a, b)=e(\mu(a, b))$.

In this section we prove that if $S \subset \mathbb{R}^{2}$ is a compact staircase-connected set, then $\xi(\cdot, \cdot)$ is an integer-valued metric. We also show some properties of this metric and prove the equivalent of the monotonicity lemma of [MP1].

Proposition 2.1. Let $S$ be a staircase-connected set in $\mathbb{R}^{2}$. Then $\xi(\cdot, \cdot)$ is an integervalued metric.

Proof. It is clear that for every two points $a, b \in S, \xi(a, b)=\xi(b, a), \xi(a, b) \geq 0$ and $\xi(a, b)=0$ if and only if $a=b$. It just remains to show the triangle inequality, that is, for every three points $a, b, c$ in $S, \xi(a, b) \leq \xi(a, c)+\xi(c, b)$.

Let $\mu(a, c)$ be a staircase with $\xi(a, c)$ edges that connects $a$ to $c$ within $S$. Let $\mu(c, b)$ be a staircase with $\xi(c, b)$ edges that connects $c$ to $b$ within $S$. Let $P$ be the concatenation of $\mu(a, c)$ and $\mu(c, b) . P$ is an orthogonal path with at most $\xi(a, c)+\xi(c, b)$ edges that connects $a$ to $b$ within $S$. Let $\mu(a, b)$ be a shortest orthogonal path from $a$ to $b$ within $S$. Clearly $e(\mu(a, b)) \leq e(P)$ and $\mu(a, b)$ is a staircase by Proposition 1.1.

Proposition 2.2. Let $S$ be a staircase-connected set in $\mathbb{R}^{2}$. Suppose $a, c \in S$ and let $b$ be a point in $S$ that belongs to the rectangle determined by a and $c$ (with sides parallel to the coordinate axes). Then $\xi(a, b) \leq \xi(a, c)$ and $\xi(b, c) \leq \xi(a, c)$.

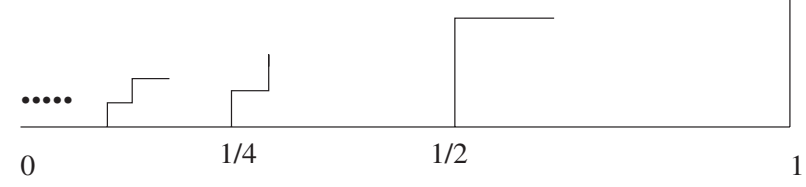

Fig. 3. A compact orthogonally connected set with infinite diameter. 
Proof. A minimal staircase $\mu(a, c)$ lies entirely within the rectangle determined by $a$ and $c$. Assume, without loss of generality, that $a$ is northwest of $c$.

If $b \in \mu(a, c)$ then clearly $\xi(a, b) \leq \xi(a, c)$.

If $b \notin \mu(a, c)$ then $b$ lies either below or above $\mu(a, c)$. If $b$ lies below $\mu(a, c)$, go from $b$ up vertically until you hit $\mu(a, c)$ at a point $b^{\prime}$, then continue from $b^{\prime}$ to $a$ on $\mu(a, c)$. This yields a staircase $P^{\prime}$ from $b$ to $a$ within $S$, and $P^{\prime}$ has at most $\xi(a, c)$ edges, since it misses entirely the last edge of $\mu(a, c)$, adjacent to $c$. (No point of the rectangle determined by $a$ and $c$ lies below that edge.) If $b$ lies above $\mu(a, c)$, go from $b$ horizontally to the left until you hit $\mu(a, c)$ at a point $b^{\prime}$, then continue to $a$ on $\mu(a, c)$. This again yields a staircase $P^{\prime}$ from $b$ to $a$ that misses the last edge of $\mu(a, c)$, and therefore has at most $\xi(a, c)$ edges.

Corollary 2.3. Under the conditions of Proposition 2.2, $\xi(a, b)+\xi(b, c)-2 \leq$ $\xi(a, c) \leq \xi(a, b)+\xi(b, c)$.

Proof. The upper bound is trivial, since $\xi(\cdot, \cdot)$ is a metric. To prove the lower bound, assume once more that $a$ is northwest of $c$.

Let $\mu(a, c)$ be a minimal staircase connecting $a$ to $c$ within $S$. If $b$ is a vertex of $\mu(a, c)$, then $\xi(a, c)=\xi(a, b)+\xi(b, c)$. If $b$ is an interior point of an edge of $\mu(a, c)$, then $\xi(a, c) \leq \xi(a, b)+\xi(b, c) \leq \xi(a, c)+1$.

If $b \notin \mu(a, c)$, then $b$ lies either below or above $\mu(a, c)$. Assume, for example, that $b$ lies below $\mu(a, c)$. The vertical line through $b\left(=L_{b}^{\mathrm{v}}\right)$ hits $\mu(a, c)$ at a point $b^{\prime}$ above $b$. The horizontal line through $b\left(=L_{b}^{\mathrm{h}}\right)$ hits $\mu(a, c)$ at a point $b^{\prime \prime}$ to the right of $b$. Since $\mu(a, c)$ is a descending path, $b^{\prime}$ precedes $b^{\prime \prime}$ on $\mu(a, c)$.

Going from $a$ to $b^{\prime}$ on $\mu(a, c)$, from $b^{\prime}$ to $b^{\prime \prime}$ through $b$ (first down, then right), and from $b^{\prime \prime}$ to $c$ on $\mu(a, c)$, we obtain a staircase $P^{\prime}$ from $a$ to $c$ via $b$ within $S$. This staircase consists of edges and parts of edges of $\mu(a, c)$, plus two edges $\left(\left[b^{\prime}, b\right]\right.$ and $\left.\left[b, b^{\prime \prime}\right]\right)$ outside $\mu(a, c)$. Thus $e\left(P^{\prime}\right) \leq 2+e(\mu(a, c))=2+\xi(a, c)$.

On the other hand, $P^{\prime}$ is a union of two subpaths: $P_{1}^{\prime}$ (from $a$ to $b$ ) and $P_{2}^{\prime}$ (from $b$ to c). Hence,

$$
e\left(P^{\prime}\right)=e\left(P_{1}^{\prime}\right)+e\left(P_{2}^{\prime}\right) \geq \xi(a, b)+\xi(b, c),
$$

ergo

$$
\xi(a, b)+\xi(b, c) \leq 2+\xi(a, c) .
$$

If $b$ lies above $\mu(a, c)$, we repeat the same procedure with $b^{\prime}$ the first point of $\mu(a, c)$ going from $b$ horizontally to the left and $b^{\prime \prime}$ the first point of $\mu(a, c)$ going from $b$ vertically down.

The staircase version of the monotonicity lemma is a straightfoward corollary of Proposition 2.2.

Corollary 2.4. Let $S$ be a staircase-connected set in $\mathbb{R}^{2}$. Let $x, y \in S$ and $b, c \in$ $\mu(x, y)$ be such that b precedes $c$ on $\mu(x, y)$. Then $\xi(x, b) \leq \xi(x, c)$. 


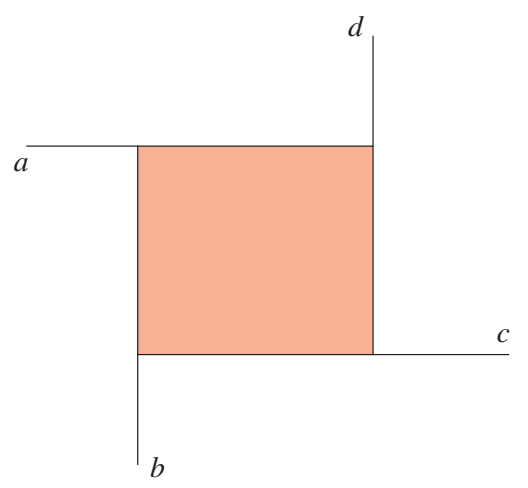

Fig. 4. Diameter 3 attained by both an ascending and a descending pair.

\section{Ascending and Descending Sets}

Definition. A pair $a, b$ of points in $\mathbb{R}^{2}\left(a=\left(a_{1}, a_{2}\right) ; b=\left(b_{1}, b_{2}\right)\right)$ is called ascending if $\left(a_{1}-b_{1}\right)\left(a_{2}-b_{2}\right)>0$. The pair $a, b$ is descending if $\left(a_{1}-b_{1}\right)\left(a_{2}-b_{2}\right)<0$.

Example 1. The set depicted in Fig. 4 is compact and staircase connected. The pair $a, c$ is descending with $\xi(a, c)=3$, and the pair $b, d$ is ascending with $\xi(b, d)=3$.

Definition. For points $a=\left(a_{1}, a_{2}\right)$ and $b=\left(b_{1}, b_{2}\right)$ in $\mathbb{R}^{2}$ we write $a \leq b$ if $a_{1} \leq b_{1}$ and $a_{2} \leq b_{2}$. (Also, $a<b$ if $a_{1}<b_{1}$ and $a_{2}<b_{2}$.)

Proposition 3.1. Let $S \subset \mathbb{R}^{2}$ be a staircase-connected set. Let $e=\left(e_{1}, e_{2}\right)$ and $f=$ $\left(f_{1}, f_{2}\right)$ be a descending pair of points of $S$. If there is a point a $\in S$ such that $a \leq e, a \leq$ $f$ (or $e \leq a, f \leq a)$, then $\xi(e, f) \leq 2$. The same holds when $e, f$ is an ascending pair, and the point a satisfies $a_{1} \geq \max \left(e_{1}, f_{1}\right), a_{2} \leq \min \left(e_{2}, f_{2}\right)\left(\right.$ or $a_{1} \leq \min \left(e_{1}, f_{1}\right)$, $\left.a_{2} \geq \max \left(e_{2}, f_{2}\right)\right)$.

Proof. Assume without loss of generality that $a \leq e, a \leq f, e_{1}<f_{1}$, and $e_{2}>f_{2}$. Let $P$ be a staircase from $a$ to $f$. Let $V$ be a vertical segment going down from $e$ to a point $e^{\prime} \in P$. Let $H$ be a horizontal segment going from $f$ to a point $v \in V$. Then $\langle f, v, e\rangle$ is a 2-staircase that connects $f$ and $e$ within $S$. (Clearly, $\xi(e, f)>1$ since $\left.e_{1} \neq f_{1}, e_{2} \neq f_{2}\right)$.

Theorem 3.2. Let $S \subset \mathbb{R}^{2}$ be a staircase-connected set. If there is an ascending (descending) pair $a, b$ of points in $S$ with $\xi(a, b) \geq 4$, then for every descending (ascending) pair e, $f$ we have $\xi(e, f)=2$.

Proof. Assume without loss of generality that $a, b$ is an ascending pair of points in $S$ $\left(a_{1}<b_{1}\right.$ and $\left.a_{2}<b_{2}\right)$.

Consider the cross $C=\left\{(x, y) \in R^{2}: a_{1} \leq x \leq b_{1} \vee a_{2} \leq y \leq b_{2}\right\}$. The complement of $C$ in $\mathbb{R}^{2}$ is the union of four open quadrants, say NE, NW, SE, SW. If $S$ meets $\mathbf{N W}$, 
then the vertex $c=\left(a_{1}, b_{2}\right)$ of $C$ is in $S$, since $S$ is staircase connected and orthogonally convex, and therefore $\xi(a, b)=2$, contrary to our assumption. Thus $\mathbf{N W} \cap S=\emptyset$. By the same argument, $\mathbf{S E} \cap S=\emptyset$.

Let $P$ be a staircase with $\xi(a, b)$ edges from $a$ to $b$ in $S$. The path $P$ divides the horizontal strip of the cross $\left(\left\{(x, y) \in R^{2}: a_{2} \leq y \leq b_{2}\right\}\right)$ into two closed half-strips: $\mathbf{E}$ (right of $P$ ) and $\mathbf{W}$ (left of $P$ ), with $\mathbf{E} \cap \mathbf{W}=P$. It also divides the vertical strip $(\{(x, y) \in$ $\left.R^{2}: a_{1} \leq x \leq b_{1}\right\}$ ) into two closed half-strips: $\mathbf{S}$ (below $P$ ) and $\mathbf{N}$ (above $P$ ), with $\mathbf{S} \cap \mathbf{N}=P$.

Let $e, f$ be a descending pair of points in $S\left(e_{1}<f_{1}, e_{2}>f_{2}\right)$. If $e \leq b$ and $f \leq b$ (or, equivalently $e, f \in \mathbf{W} \cup \mathbf{S W} \cup \mathbf{S}$ ), then $\xi(e, f)=2$ by Proposition 3.1. The same holds when $a \leq e, a \leq f$ (i.e., $e, f \in \mathbf{E} \cup \mathbf{N E} \cup \mathbf{N}$ ).

This covers all cases when one of the points $e, f$ does not belong to the cross $C$, or when both $e$ and $f$ belong to the same half-strip, or to adjacent half-strips ( $\mathbf{E}$ and $\mathbf{N}$, or $\mathbf{W}$ and $\mathbf{S}$ ).

There remains the case where $e$ and $f$ belong to opposite half-strips ( $\mathbf{E}$ and $\mathbf{W}$, or $\mathbf{N}$ and $\mathbf{S}$ ).

Assume, without loss of generality, that $e \in \mathbf{W}$ and $f \in \mathbf{E}$. Since they do not belong to two adjacent half-strips, none of them lies within the central rectangle $[a, d, b, c]$. Draw a horizontal segment $H$ from $e$ to $P$, and another horizontal segment $H^{\prime}$ from $f$ to $P$. $H$ meets $[a, c]$ at the point $\left(a_{1}, e_{2}\right) \in S$, and $H^{\prime}$ meets $[d, b]$ at $\left(b_{1}, f_{2}\right) \in S$. Since $f_{2}<e_{2} \leq b_{2},\left(b_{1}, e_{2}\right)$ is also in $S$ (vertical convexity). Thus $\left\langle a,\left(a_{1}, e_{2}\right),\left(b_{1}, e_{2}\right), b\right\rangle$ is a 3 -staircase connecting $a$ to $b$ within $S$, which contradicts our assumption that $\xi(a, b) \geq 4$.

In view of Theorem 3.2, we divide the staircase-connected sets satisfying $\operatorname{stdiam}(S)=$ $k \geq 4$ into two disjoint classes: ascending sets and descending sets. $S$ is ascending (descending) if it includes an ascending (descending) pair $a, b$ with $\xi(a, b) \geq 3$.

Reflection in the $x$-axis (or $y$-axis) transforms ascending sets into descending sets and vice versa.

\section{Staircase Stars and Kernels}

Let $S \subset \mathbb{R}^{2}$ be a staircase-connected set. We define the staircase $r$-star of a point $z$ in $S\left(s s t_{r}(z, S)\right)$ to be the set of all points $y \in S$ which can be joined to $z$ within $S$ by an $r$-staircase, i.e., by a staircase of at most $r$ edges. The intersection of all staircase $r$-stars is called the staircase $\boldsymbol{r}$-kernel of $S$ and denoted by $\operatorname{Ker}_{r}(S)$.

Assume $\operatorname{stdiam}(S)=k$. If $r<k / 2$ then clearly $\operatorname{Ker}_{r}(S)=\emptyset$. If $r=k / 2$ then $\operatorname{Ker}_{r}(S)$ may or may not be empty, as shown in the examples in Fig. 5.

In the first example, $k=4, r=2$ and $\operatorname{Ker}_{2}(S)$ consists of two points, $a$ and $b$. In the second example, $k=4, r=2$ and $\operatorname{Ker}_{2}(S)$ is empty.

For $S \subset \mathbb{R}^{2}$ compact and staircase connected, we will show that if $r>k / 2$ then $\operatorname{Ker}_{r}(S) \neq \emptyset$. We will also show that if $r \geq k / 2+1$ then $\operatorname{Ker}_{r}(S)$ is again compact and staircase connected.

When $r=k / 2+1 / 2, \operatorname{Ker}_{r}(S)$ need not be staircase connected, but it is connected, in fact, connected by monotone paths (see [MP2]).

Example 2. Take a rectangle $R$ with sides parallel to the coordinate axes, whose length 

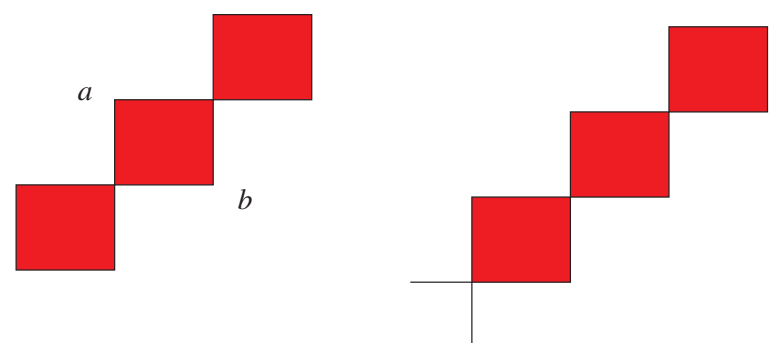

Fig. 5. Two sets of diameter 4 . The second kernel is nonempty on the left, empty on the right.

is $2 \kappa$ times larger than its height ( $\kappa$ a positive integer). Tilt $R$ by $45^{\circ}$. This yields a compact staircase-connected set $S$ with $\operatorname{stdiam}(S)=2 \kappa+1$. The staircase $(\kappa+1)$-kernel of $S$ is just the segment that joins the midpoints of the long sides of $S$. Thus, $\operatorname{Ker}_{\kappa+1}(S)$ is not staircase connected (though it is connected).

Lemma 4.1. Let $S$ be a staircase-connected set in $\mathbb{R}^{2}$, let $r$ be any positive integer and let $x$ be any point of $S$. Then $\operatorname{sst}_{r}(x, S)$ is orthogonally convex.

Proof. Suppose $[y, z]$ is a horizontal or vertical segment with endpoints in $\operatorname{sst}_{r}(x, S)$. We must show that $[y, z] \subset \operatorname{sst}_{r}(x, S)$.

Assume, without loss of generality, that $[y, z]$ is horizontal and that $y_{1}<z_{1}, y_{2}=$ $z_{2}=h$. Take a point $u=\left(u_{1}, h\right) \in[y, z]$. We must show that $\xi(u, x) \leq r$. If $x_{1} \leq u_{1}$, then $\xi(x, u) \leq \xi(x, z) \leq r$ by Proposition 2.2. If $x_{1} \geq u_{1}$, then $\xi(x, u) \leq \xi(x, y) \leq r$, again by Proposition 2.2.

A similar result, valid for simply connected orthogonal polygons, has been proved by Topala in [T]. These sets have also been investigated by Breen in [B1] and [B2].

Corollary 4.2. Let $S$ be a staircase-connected set in $\mathbb{R}^{2}$, let $r$ be any positive integer and let $x$ be any point of $S$. Then $\operatorname{sst}_{r}(x, S)$ is staircase connected.

Proof. This corollary is an easy consequence of Proposition 1.1 and Lemma 4.1.

Lemma 4.3. Let $S$ be a compact staircase-connected set in $\mathbb{R}^{2}$, let $r$ be any positive integer and let $x$ be any point of $S$. Then $s_{s} t_{r}(x, S)$ is compact.

Proof. Since $S$ is compact, it suffices to show $\operatorname{sst}_{r}(x, S)$ is closed. Consider an infinite sequence of points $\left\{q_{i}\right\}_{i=1}^{\infty}$ in $s t_{r}(x, S)$ that converges to a point $q$ in $S$. We need to show that $q \in \operatorname{sst}_{r}(x, S)$, i.e., there exists a staircase path in $S$ of at most $r$ edges joining $x$ and $q$.

Denote by $L_{x}^{\mathrm{h}}$ and $L_{x}^{\mathrm{v}}$ the horizontal and vertical lines through $x$, respectively. If $q$ belongs to any of these two lines, then $[x, q] \subset S$, since $S$ is orthogonally convex, and the claim follows. If not, then $q$ lies in the interior of one of the four quadrants determined by $L_{x}^{\mathrm{h}}$ and $L_{x}^{\mathrm{v}}$. Assume without loss of generality that $q$ and $\left\{q_{i}\right\}_{i=1}^{\infty}$ are interior points of the northeast quadrant, and also that $\xi\left(x, q_{i}\right)=r$ for every $i$. If so, for every $q_{i}$ there exists an 
$r$-staircase $\mu\left(x, q_{i}\right)=\left\langle x=p_{0}^{i}, p_{1}^{i}, \ldots, p_{r}^{i}=q_{i}\right\rangle$ joining $x$ to $q_{i}$ via $S$. Without loss of generality, assume that all the segments $\left[x, p_{1}^{i}\right]$ are horizontal, that is, the vectors induced by these segments have direction west to east. It follows that every segment $\left[p_{j}^{i}, p_{j+1}^{i}\right]$ with $j$ even is horizontal, while every segment $\left[p_{j}^{i}, p_{j+1}^{i}\right]$ with $j$ odd is pointing upward. By passing to a subsequence if necessary, we may assume that for every $j(0 \leq j \leq r)$, the sequence $\left\{p_{j}^{i}\right\}_{i=1}^{\infty}$ converges to a point $p_{j}$ in $S$. Then every sequence of horizontal (or vertical) segments $\left[p_{j}^{i}, p_{j+1}^{i}\right]$ will converge to the horizontal (or vertical) segment $\left[p_{j}, p_{j+1}\right]$ (which may degenerate to a point). The path $\left\langle x=p_{0}, \ldots, p_{r}=q\right\rangle$ is a staircase path in $S$ with at most $r$ edges joining $x$ and $q$, and our claim is proved.

Corollary 4.4. Let $S$ be a compact staircase-connected set in $\mathbb{R}^{2}$, then for every positive integer $r, \operatorname{Ker}_{r}(S)$ is compact.

We define the staircase radius of $S(\operatorname{strad}(S))$ to be the smallest integer $r$ such that $\operatorname{Ker}_{r}(S) \neq \emptyset$.

Theorem 4.5. If $S \subset \mathbb{R}^{2}$ is a compact staircase-connected set, and $\operatorname{stdiam}(S)=k \geq 1$, then

$$
\operatorname{strad}(S)= \begin{cases}k / 2 \text { or } k / 2+1, & k \text { even }, \\ (k+1) / 2, & k \text { odd } .\end{cases}
$$

Proof. If $k=1$ then $S$ is a line segment (horizontal or vertical). If $k=2$ then $\operatorname{strad}(S)$ may be either 1 (only when $S$ is a union of a horizontal segment and a vertical segment, with a common point) or 2 .

Assume, from now on, that $k \geq 3$. Let $\left[a_{1}, c_{1}\right]$ and $\left[b_{2}, d_{2}\right]$ be the projections of $S$ onto the first and second coordinates, respectively. (Clearly $-\infty<a_{1}<c_{1}<\infty$, $-\infty<b_{2}<d_{2}<\infty$.) $R(S)=\left[a_{1}, c_{1}\right] \times\left[b_{2}, d_{2}\right]$ is the smallest rectangle with sides parallel to the coordinate axes that includes $S$.

Define

$$
\begin{aligned}
& I_{1}=\left[\left(a_{1}, a_{2}^{\prime}\right),\left(a_{1}, a_{2}\right)\right]=\left[a^{\prime}, a\right], \\
& I_{2}=\left[\left(b_{1}^{\prime}, b_{2}\right),\left(b_{1}, b_{2}\right)\right]=\left[b^{\prime}, b\right], \\
& I_{3}=\left[\left(c_{1}, c_{2}\right),\left(c_{1}, c_{2}^{\prime}\right)\right]=\left[c, c^{\prime}\right], \\
& I_{4}=\left[\left(d_{1}, d_{2}\right),\left(d_{1}^{\prime}, d_{2}\right)\right]=\left[d, d^{\prime}\right]
\end{aligned}
$$

to be the intersections of $S$ with the left edge, bottom edge, right edge and top edge of $R(S)$, respectively $\left(a_{2}^{\prime} \leq a_{2}, b_{1}^{\prime} \leq b_{1}, c_{2} \leq c_{2}^{\prime}, d_{1} \leq d_{1}^{\prime}\right)$.

If $\left[a_{2}^{\prime}, a_{2}\right] \cap\left[c_{2}, c_{2}^{\prime}\right] \neq \emptyset$ then $S$ has a horizontal transversal $T=\left[\left(a_{1}, y\right),\left(c_{1}, y\right)\right]$ $\left(y \in\left[a_{2}^{\prime}, a_{2}\right] \cap\left[c_{2}, c_{2}^{\prime}\right]\right)$. In this case every point of $S$ can be joined to $T$ by a vertical segment in $S$, and therefore every point of $S$ can be joined to any point of $T$ by an orthogonal path with at most two edges in $S$. It follows that $\operatorname{stdiam}(S) \leq 3, \operatorname{strad}(S) \leq 2$. Since we assume that $k>2$ we obtain $\operatorname{stdiam}(S)=3, \operatorname{strad}(S)=2$.

Assume, from now on, that $\left[a_{2}^{\prime}, a_{2}\right] \cap\left[c_{2}, c_{2}^{\prime}\right]=\emptyset$. Similarly, assume that $\left[b_{1}^{\prime}, b_{1}\right] \cap$ $\left[d_{1}, d_{1}^{\prime}\right]=\emptyset$. Thus $a_{2}<c_{2}$ or $a_{2}^{\prime}>c_{2}^{\prime}$. Assume without loss of generality that $a_{2}<c_{2}$ 


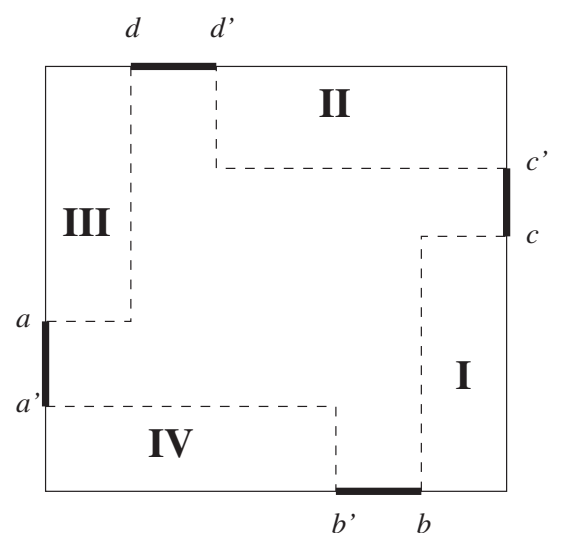

Fig. 6. Case I.

(otherwise reflect $S$ in the $y$-axis). As for the horizontal sides, we have either $b_{1}<d_{1}$ or $b_{1}^{\prime}>d_{1}^{\prime}$.

Case I: $b_{1}^{\prime}>d_{1}^{\prime}$. (See Fig. 6.) We show that in this case, $\operatorname{stdiam}(S) \leq 3$ and $\operatorname{strad}(S) \leq 2$.

Consider the points $a^{\prime}, b^{\prime}$. They are a descending pair and satisfy $a^{\prime}<c, b^{\prime}<c$. By Proposition 3.1, they are connected in $S$ by a 2-staircase with midvertex $\left(b_{1}^{\prime}, a_{2}^{\prime}\right)$. By the same token, we find a 2-staircase in $S$ that connects $d^{\prime}$ to $c^{\prime}$ with midvertex $\left(d_{1}^{\prime}, c_{2}^{\prime}\right)$; a 2-staircase in $S$ that connects $b$ to $c$ with midvertex $\left(b_{1}, c_{2}\right)$; and, finally, a 2-staircase in $S$ that connects $a$ to $d$ with midvertex $\left(d_{1}, a_{2}\right)$.

These four 2-paths, together with the four (possibly degenerate) segments $I_{1}, I_{2}, I_{3}, I_{4}$, bound a rectangle $D=\left[d_{1}, b_{1}\right] \times\left[a_{2}^{\prime}, c_{2}^{\prime}\right]$ with four rectangular protrusions, as shown in Fig. 6. This central figure, call it $E$, is certainly part of $S$.

$R(S) \backslash E$ consists of four pairwise disjoint rectangles, numbered I, II, III and IV (see Fig. 6).

We urge the reader to verify the following claims:

1. Each two points of $E$ can be joined by a staircase path with at most three edges. Three edges are actually needed only to connect points in opposite protrusions.

2. Every point of $S$ in one of the rectangles I, II, III, IV can be joined to any point of $E$ by a staircase path with at most three edges.

3. Every two points in the same rectangle, say I, can be joined by a staircase path with at most two edges in $S$.

4. Every two points of $S$ in adjacent rectangles (say I and II) can be joined by a staircase path with at most three edges in $S$.

5. Every two points of $S$ in opposite rectangles (say I and III) can be joined by a staircase path with at most three edges in $S$.

6. Every point of $S$ can be joined to any point of $D$ by a staircase path with at most two edges in $S$.

It follows that $\operatorname{strad}(S)=2, \operatorname{stdiam}(S)=3$. 
Case II: $b_{1}<d_{1}$. As in Case I, consider the points $b$ and $c$. $S$ includes an ascending staircase path $P$ from $b$ to $c$ with $e(P)=\xi(b, c)$. This path must start with a vertical edge and end with a horizontal edge. Therefore, it must have an even number of edges, say $2 \kappa$. Denote by $w$ the middle vertex of $P$. We claim that every point of $S$ (say $z=\left(z_{1}, z_{2}\right)$ ) can be joined to $w$ by a staircase path of at most $\kappa+1$ edges in $S$.

If $z_{2} \leq c_{2}$, move from $z$ horizontally until you hit $P$. Then continue on $P$ towards $w$. This yields an orthogonal path of at most $\kappa+1$ edges from $z$ to $w$ within $S$. If this path is not monotone, i.e., not a staircase path, then it can be shortened by suitable shortcuts until it becomes a staircase path (within $S$ ) from $z$ to $w$ (see Proposition 1.1).

If $z_{1} \geq b_{1}$, we proceed in the same way, starting with a vertical move from $z$ to $P$.

There remains the case $z_{1}<b_{1}, z_{2}>c_{2}$. The case where some point $z \in S$ satisfies this condition will be treated below.

If there are no points $z \in S$ with $z_{1}<b_{1}, z_{2}>c_{2}$, then $w \in \operatorname{Ker}_{\kappa+1}(S)$, hence $\operatorname{strad}(S) \leq \kappa+1$. Clearly, $\operatorname{strad}(S) \geq \frac{1}{2} \cdot \operatorname{stdiam}(S) \geq \frac{1}{2} \cdot \xi(b, c)=\kappa$. Therefore, the possible values for radius and diameter are:

$$
\begin{aligned}
& \operatorname{strad}(S)=\kappa, \quad \operatorname{stdiam}(S)=2 \kappa, \\
& \operatorname{strad}(S)=\kappa+1, \quad \operatorname{stdiam}(S)=2 \kappa, \\
& \operatorname{strad}(S)=\kappa+1, \quad \operatorname{stdiam}(S)=2 \kappa+1, \\
& \operatorname{strad}(S)=\kappa+1, \quad \operatorname{stdiam}(S)=2 \kappa+2,
\end{aligned}
$$

and our claim holds.

If there is a point $z \in S$ with $z_{1}<b_{1}, z_{2}>c_{2}$, then, by Proposition 3.1, $\xi(b, c)=$ $2 \kappa=2$, i.e., $\kappa=1$. Therefore, $w=\left(b_{1}, c_{2}\right)$.

Applying the same proposition again to the descending pair $z, w$ (with $z \leq d, w \leq d$ ), we find that $\xi(z, w)=2$. Thus $w \in \operatorname{Ker}_{2}(S)$, hence $\operatorname{strad}(S)=2$. In this case, $\operatorname{stdiam}(S)$ may be 3 or 4 , as shown in Fig. 7. $(\operatorname{stdiam}(S)>2$ since $\xi(a, c)=3$.)

If we drop the compactness of $S$ in the assumptions of Theorem 4.5, then $\operatorname{Ker}_{(k+1) / 2}(S)$ may be empty, as shown in the following example:
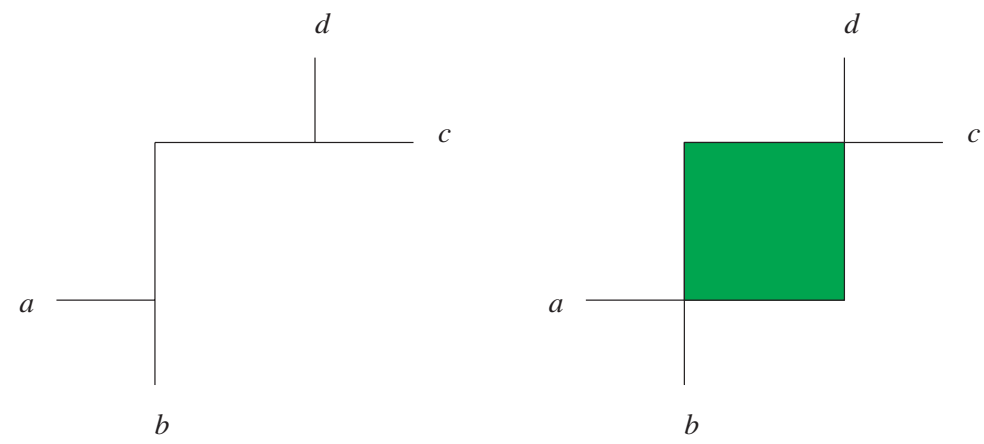
Example 3. Consider the tilted rectangle $R$ from Example 2. Erase from its boundary both (closed) long sides, and one of the short sides. This yields a set $S \subset \mathbb{R}^{2}$ with $\operatorname{stdiam}(S)=2 \kappa+1$ and whose staircase $(\kappa+1)$-kernel is empty.

The correct version of Theorem 4.5 without compactness is as follows:

Theorem 4.6. If $S \subset \mathbb{R}^{2}$ is a staircase-connected set, $\operatorname{stdiam}(S)=k \geq 1$, then

$$
\operatorname{strad}(S)= \begin{cases}k / 2 \text { or } k / 2+1, & k \text { even }, \\ (k+1) / 2 \text { or }(k+3) / 2, & k \text { odd } .\end{cases}
$$

Proof. The inequalities $\operatorname{strad}(S) \leq \operatorname{stdiam}(S) \leq 2 \cdot \operatorname{strad}(S)$ hold without assuming compactness. Thus all we have to show is that

$$
\operatorname{strad}(S) \leq \frac{1}{2} \cdot \operatorname{stdiam}(S)+\frac{3}{2} .
$$

This is trivial for $\operatorname{stdiam}(S)=k \leq 3$. Assume therefore $k \geq 4$ and suppose, without loss of generality, that $S$ is ascending.

Define, for any point $y \in S$,

$$
\begin{gathered}
\alpha(y)=\max \{\xi(x, y): x \in S, x \leq y\}, \\
\beta(y)=\max \{\xi(y, z): z \in S, y \leq z\} .
\end{gathered}
$$

These functions have the following properties, whose proofs are straightfoward:

(A) $\alpha$ is nondecreasing; $\beta$ is nonincreasing. That is, if $y, y^{\prime} \in S, y \leq y^{\prime}$, then $\alpha(y) \leq \alpha\left(y^{\prime}\right)$ and $\beta\left(y^{\prime}\right) \leq \beta(y)$.

(B) If $y, y^{\prime} \in S, y \leq y^{\prime}$ and the segment $\left[y, y^{\prime}\right]$ is either horizontal or vertical, then $\alpha\left(y^{\prime}\right) \leq \max \{2,1+\alpha(y)\}$. Thus $\alpha\left(y^{\prime}\right) \leq 1+\alpha(y)$ provided $\alpha(y) \geq 1$. Similarly, $\beta(y) \leq \max \left\{2,1+\beta\left(y^{\prime}\right)\right\}$

(C) For all $y \in S, \alpha(y)+\beta(y) \leq 2+\operatorname{stdiam}(S)$, by Corollary 2.3 .

(D) If $\operatorname{stdiam}(S)=k \geq 4$ and $S$ is ascending, then $\operatorname{strad}(S) \leq r$ iff $\alpha(y) \leq r$ and $\beta(y) \leq r$ for some $y \in S$.

Now we turn to the proof of $(*)$. Choose two points $a, b \in S$ such that $\xi(a, b)=$ $\operatorname{stdiam}(S)=k$. Since $S$ is ascending, we may assume $a \leq b$. Let $\left\langle a=x_{0}, \ldots, x_{k}=b\right\rangle$ be a $k$-staircase in $S$ from $a$ to $b$. Note that $\alpha\left(x_{0}\right) \leq 2, \beta\left(x_{0}\right)=k \geq 4, \alpha\left(x_{k}\right)=k \geq 4$, $\beta\left(x_{k}\right) \leq 2$. Let $i$ be the last index for which $\alpha\left(x_{i}\right) \leq \beta\left(x_{i}\right)$. Thus $\alpha\left(x_{i+1}\right)>\beta\left(x_{i+1}\right)$.

If $(*)$ is false, then, by (D), $\beta\left(x_{i}\right) \geq \frac{1}{2} k+2$ and therefore, by (C),

$$
\alpha\left(x_{i}\right) \leq \frac{1}{2} k .
$$

By the same token, if $(*)$ is false then

$$
\alpha\left(x_{i+1}\right) \geq \frac{1}{2} k+2 \geq 4 .
$$

Inequalities $(* *)$ and $(* * *)$ together contradict $(\mathrm{B})$. 
Theorem 4.7. Let $S \subset \mathbb{R}^{2}$ be orthogonally convex, and let $\operatorname{stdiam}(S)=k$. Assume $k / 2+1 \leq r \leq k$, and suppose $a, c \in \operatorname{Ker}_{r}(S)$. Then:

1. If $\xi(a, c) \neq 2$, then every point $b$ of $S$ that lies in the rectangle determined by $a$ and $c$ also belongs to $\operatorname{Ker}_{r}(S)$. (In particular, every staircase from a to $c$ within $S$ lies in $\operatorname{Ker}_{r}(S)$.)

2. If $\xi(a, c)=2$ then there exists a 2-staircase $v(a, c)$ from a to $c$ in $\operatorname{Ker}_{r}(S)$.

Note that if $r=k$, then $\operatorname{Ker}_{r}(S)=S$ and the theorem does not say anything new. We may therefore assume that $k / 2+1 \leq r<k$. This condition implies $k \geq 4$ and $r \geq 3$.

Proof. Since $k \geq 4$, we may assume without loss of generality that the set $S$ is ascending (Theorem 3.2).

(1) If $\xi(a, c)=1$ then $b \in \operatorname{Ker}_{r}(S)$ by Lemma 4.1. So we may assume $\xi(a, c) \geq 3$, and therefore $a, c$ is an ascending pair. If $x \in S$ is southeast or northwest of $b$, then $\xi(b, x) \leq 2$, since $S$ is ascending. If $x$ is northeast of $b$, then $\xi(b, x) \leq \xi(a, x) \leq r$, by the rectangle property (Proposition 2.2). If $x$ is southwest of $b$, then $\xi(b, x) \leq \xi(c, x) \leq r$, again by the rectangle property.

(2) Let $\langle a, q, c\rangle$ be a 2-staircase path in $S$ from $a$ to $c$. If the pair $a, c$ is ascending, then $q \in \operatorname{Ker}_{r}(S)$, and therefore $\langle a, q, c\rangle \subset \operatorname{Ker}_{r}(S)$. This is proved exactly as in (1).

Assume then that the pair $a, c$ is descending (with $c$ southeast of $a$ ), and assume without loss of generality that $q$ is the intersection point of the lines $L_{a}^{\mathrm{h}}$ (horizontal through $a$ ) and $L_{c}^{\mathrm{v}}$ (vertical through $c$ ). If $q \in \operatorname{Ker}_{r}(S)$, we are done. If $q \notin \operatorname{Ker}_{r}(S)$, then there exists a point $q^{\prime}$ in $S$ such that $\xi\left(q, q^{\prime}\right)>r$. If $q^{\prime}$ lies above $L_{a}^{\mathrm{h}}$ (including $\left.L_{a}^{\mathrm{h}}\right)$ then $\xi\left(q^{\prime}, q\right) \leq \xi\left(q^{\prime}, c\right) \leq r$. If $q^{\prime}$ lies to the right of $L_{c}^{\mathrm{v}}$ (including $L_{c}^{\mathrm{v}}$ ) then $\xi\left(q^{\prime}, q\right) \leq \xi\left(q^{\prime}, a\right) \leq r$. If $q^{\prime}$ lies between $L_{a}^{\mathrm{h}}$ and $L_{c}^{\mathrm{h}}$ (including both lines) then $\xi\left(q^{\prime}, q\right) \leq 2 \leq r$. The same holds when $q^{\prime}$ lies between $L_{a}^{\mathrm{v}}$ and $L_{c}^{\mathrm{v}}$ (including both lines).

We conclude that $q^{\prime}$ is strictly southwest of $p$ (the intersection point of $L_{a}^{\mathrm{v}}$ and $L_{c}^{\mathrm{h}}$ ). This implies that $p \in S$ as well (since $S$ is staircase connected) and therefore the whole rectangle $[a, p, c, q]$ is in $S$.

If $p \in \operatorname{Ker}_{r}(S)$, we are done. Otherwise there is a point $p^{\prime} \in S$ with $\xi\left(p, p^{\prime}\right)>r$. Repeating the previous argument, we find that $p^{\prime}$ is strictly northeast of $q$.

Here $\xi\left(q, q^{\prime}\right)>r \geq \xi\left(q^{\prime}, a\right)$. Since $a$ and $q$ are adjacent, we find that $\xi\left(q, q^{\prime}\right)=r+1$, $\xi\left(q^{\prime}, a\right)=r$. Similarly $\xi\left(q^{\prime}, c\right)=r, \xi\left(p, p^{\prime}\right)=r+1, \xi\left(p^{\prime}, a\right)=r$ and $\xi\left(p^{\prime}, c\right)=r$.

Now consider the distance $\xi\left(p^{\prime}, q^{\prime}\right)$. Since $a$ and $c$ lie between $p^{\prime}$ and $q^{\prime}$ (i.e., $a$ and $c$ belong to the rectangle determined by $p^{\prime}$ and $q^{\prime}$ ), we find that

$$
k \geq \xi\left(p^{\prime}, q^{\prime}\right) \geq \xi\left(p^{\prime}, a\right)+\xi\left(a, q^{\prime}\right)-2=2 r-2
$$

by Corollary 2.3.

In the theorem we assume that $k / 2+1 \leq r$, hence $k \leq 2 r-2$. Thus $k=2 r-2$ and the inequalities in $(\circledast)$ hold as equalities.

Let us draw a $k$-staircase $Q$ from $q^{\prime}$ to $p^{\prime}$. $Q$ starts with a horizontal edge and ends with a vertical edge, or vice versa. Both cases are essentially the same. In Fig. 8, the first edge is horizontal and $r$ is odd (actually, $r=7$ ). If $r$ is even then the points $z$ and $w$ in Fig. 8 exchange roles, and some of the wording below must be adjusted accordingly. 


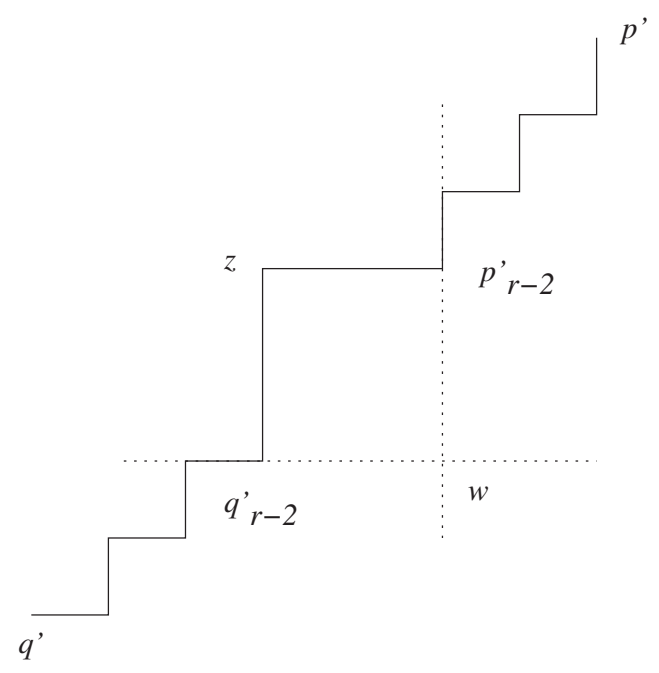

Fig. 8. $k$-Staircase from $q^{\prime}$ to $p^{\prime}$ in $S$.

Denote the vertices of $Q$ by $\left(q^{\prime}=\right) q_{0}^{\prime}, q_{1}^{\prime}, \ldots, q_{r-2}^{\prime}, z, p_{r-2}^{\prime}, \ldots, p_{0}^{\prime}\left(=p^{\prime}\right)$. If $a$ lies on the line $L_{z}^{\mathrm{v}}$ or to its left, then $\xi\left(q^{\prime}, a\right) \leq r-1$. If $a$ lies on, or below the line $L_{w}^{\mathrm{h}}$, then $\xi\left(q^{\prime}, a\right) \leq r-2$. If $a$ lies on, or above the line $L_{z}^{\mathrm{h}}$, then $\xi\left(p^{\prime}, a\right) \leq r-1$. If $a$ lies on the line $L_{w}^{\mathrm{v}}$ or to its right, then $\xi\left(p^{\prime}, a\right) \leq r-2$. The same holds for $c$ instead of $a$.

It follows that both $a$ and $c$ lie in the interior of the rectangle $\left[p_{r-2}^{\prime}, w, q_{r-2}^{\prime}, z\right]$. However, this implies that the points $p$ and $q$ lie in the same rectangle and therefore $\xi\left(p, p^{\prime}\right) \leq \xi\left(p^{\prime}, q_{r-2}^{\prime}\right)=r$ and $\xi\left(q, q^{\prime}\right) \leq \xi\left(q^{\prime}, p_{r-2}^{\prime}\right)=r$, a contradiction.

The following theorem is an immediate consequence of Theorem 4.7:

Theorem 4.8. Let $S \subset \mathbb{R}^{2}$ be compact, orthogonally convex and let $\operatorname{stdiam}(S)=k$. If $k / 2+1 \leq r \leq k$, then $\operatorname{Ker}_{r}(S)$ is also a compact staircase-connected set.

\section{Acknowledgements}

We thank Mr. Shlomi Kotler and Miss Rosi Milgrom for their technical support.

\section{References}

[B1] Breen, M., Staircase kernels in orthogonal polygons, Arch. Math. 59 (1992), 588-594.

[B2] Breen, M., Staircase $k$-kernels for orthogonal polygons, Arch. Math. 63 (1994), 182-190.

[MP1] Magazanik, E., and Perles, M.A., Generalized convex kernels of simply connected $L_{n}$ sets in the plane, accepted for publication in Israel J. Math.

[MP2] Magazanik, E., and Perles, M.A., Staircase kernels, accepted for publication in Adv. Geom.

[T] Topala, O., On intersection of maximal orthogonally $k$-starshaped polygons, Geom.Dedicata 78 (1999), 271-278.

Received November 8, 2005, and in revised form October 2, 2006. Online publication April 18, 2007. 